\title{
COMMUNICATION
}

\section{Molecular inversion probe-based SPR biosensing for specific, label-free and real-time detection of regional DNA methylation ${ }^{\dagger}$}

Received ooth January 2013,

Accepted ooth January 2013

DOI: $10.1039 /$ xoxxooooox

www.rsc.org/

\author{
Laura G. Carrascosa, ${ }^{a} *$ Abu Ali Ibn Sina, ${ }^{a}$ Ramkumar Palanisamy, ${ }^{a}$ Borja Sepulveda, ${ }^{c}$ \\ Marinus A. Otte, ${ }^{c}$ Sakandar Rauf, ${ }^{a}$ Muhamad J. A. Shiddiky and Matt Trau ${ }^{a}{ }^{a}{ }_{*}$
}

DNA methylation has the potential to be a clinically important biomarker in cancer. This communication reports a real-time and label-free biosensing strategy for DNA methylation detection in cancer cell line. This has been achieved by using surface Plasmon resonance biosensing combined with the highly specific molecular inversion probe based amplification method, which requires only $50 \mathrm{ng}$ of bisufite treated genomic DNA.

DNA methylation is the process where a methyl group is added to the carbon-5 position of cytosine in a CpG dinucleotide ${ }^{1}$. Differences in DNA methylation levels between normal and cancer cells have been proposed as biomarkers to retrieve clinically relevant information regarding the stage of disease or cancer subtype, which in turn might help to underpin prognosis and appropriate mode of treatment. ${ }^{2}$ Much attention has been focused on the detection of DNA methylation using different sensors schemes, ${ }^{3-9}$ however most widely used techniques for DNA methylation are bisulfite sequencing $^{10}$ and affinity-enrichement. ${ }^{5}$ Bisulfite-sequencing is a very sensitive technique capable of retrieving single cytosine methylation information, but the methodology itself is complex and prone to DNA amplification errors. Detecting a series of several CpG sites in a region (e.g., the promoter region) has the potential to extract the desired methylation information without requiring single $\mathrm{CpG}$ site resolution. In this context, affinity based methods using methyl binding domain proteins that specifically recognize methylated-CpG rich regions, offer a better alternative. While all these methods have excellent analytical performances in detecting regional DNA methylation, they are poorly suited for routine diagnostics due to the high running cost, long assay time, complicated chemistries and detection procedures.

Surface Plasmon resonance (SPR) is one of the most powerful alternative analytical methods for circumventing these type of problems,

${ }^{a}$ Australian Institute for Bioengineering and Nanotechnology (AIBN), The University of Queensland, Brisbane, QLD 4072, Australia. ${ }^{b}$ School of Chemistry and Molecular Biosciences, University of Queensland, Brisbane, QLD 4072, Australia. ${ }^{C}$ Catalan Institute of Nanoscience and Nanotechnology (ICN2), CSIC \& CIBER-BBN. ICN2 Building, Campus UAB 08193 Bellaterra, Spain E-mails: lgcarrascosa@uq.edu.au and m.trau@uq.edu.au. 广Electronic Supporting Information (ESI): Experimental details as noted in text, see DOI: 10.1039/c000000x/

while providing label-free, real-time, reproducible and sensitive biomolecular detection. ${ }^{11}$ In SPR biosensing, the binding of the target analyte to its surface-bound receptor counterpart produces a local change in refractive index over time at the sensing surface, which is quantitative with the relative mass increase associated with target capture, thus enabling the real-time and label-free readout of these targets ${ }^{12}$. This method has previously been used to detect regional DNA methylation using proteins with affinity to $\mathrm{CpG}$ rich regions. ${ }^{6,9}$ However, the reproducibility of affinity based methods depends on antibody specificity and CG-density. ${ }^{13}$

Herein, we report a novel strategy which synergistically couples the label-free, real-time nature of the SPR biosensor with the sensitive, specific and multiplexing capability of the molecular inversion probes (MIPs) for accurate detection of regional DNA methylation. The principle involves utilizing a MIP, which is a single stranded oligonucleotide (ss-oligo) capable of hybridizing to a genomic DNA target by two inverted recognition ends. ${ }^{14,15}$ MIPs are well-known for their capability for deciphering genetic ${ }^{16}$ and epigenetic ${ }^{17,18}$ information from multiple regions across the genome in a highly specific and sensitive way. MIP binding to the single stranded-DNA generates a gap of one or several bases long between the hybridized recognition ends. Following the gap-filling and enzymatic ligation, a circularized DNA probe carrying the imprinted information from the interrogated region is generated (for details, see Fig $\mathrm{S}_{1}, \mathrm{ESI}$ )). PCR amplification of the circularized MIPs employing universal primers enables detecting the amplified-amplicons using DNA array platform ${ }^{15}$ or beads. ${ }^{18}$ The superior specificity and accuracy of MIP based approches over conventional primer binding approaches can be attributed to the enzymatic digestion of all non-circularized probes and use of the universal primers for ampliflying all MIPs. This, in combination with SPR has the potential to sensitively and specifically measure regional DNA methylation in real-time and label-free manner, while avoiding the use of affinity based proteins.

Scheme 1 outlines a strategy to combine MIPs and SPR for regional methylation detection of a cluster of eight $\mathrm{CpG}$ sites located within a span of 53 bases downstream of the transcription start site of the engrailed (En1) gene (Fig. $\mathrm{S}_{1}$ and $\mathrm{S}_{2}, \mathrm{ESI}+$ ). The methylation status of this region has been recently highlighted as a potential biomarker in several cancer types. ${ }^{19}$ According to our sequencing data (Fig. S2, ESI†), this region is also 


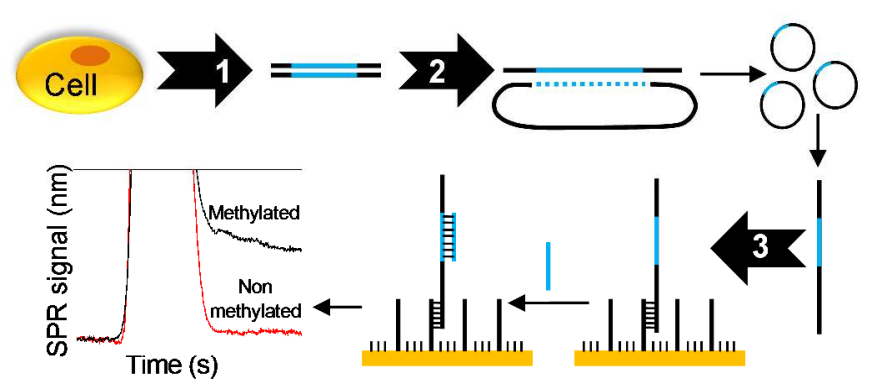

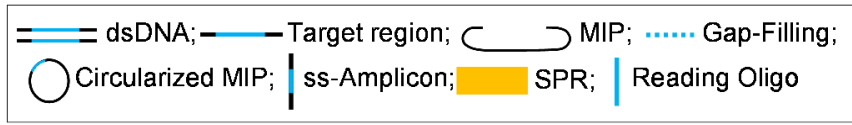

Scheme 1. Methodological approach for the label-free and real-time detection of regional DNA methylation based on MIP and SPR. Inset, typical SPR sensograms for the reading oligo step hybridized with methylated and unmethylated targets.

methylated in $\mathrm{MCF} 7$ breast cancer cells, thus we selected this cell type as a source for methylated DNA. For unmethylated DNA, we used whole genome amplified (WGA) DNA. ${ }^{20}$ The assay consisted of three steps: $(i)$ DNA extraction and bisulfite treatment to convert the epigenetic change into base changes, (ii) DNA interrogation by MIPs followed by the generation of single stranded-DNA amplicons using asymmetric PCR and (iii) reading the methylation status of amplicons with SPR using a sandwich approach (see ESIt for details).

Briefly, after extraction from cell lines (Scheme 1, step 1), genomic DNA was bisulfite treated enabling the selective conversion of unmetylated cytosines into uracile while leaving methylated ones unchanged. Then, the region of interest was interrogated with a MIP using a fill-in approach. In the fill-in approach, the MIP binds to the DNA, and generates a broad gap of several bases (i.e. 53 bases) between the MIP recognition ends, which represents the methylated region to be interrogated. This gap was then filled by a polymerase and subsequently ligated by a ligase, leading to a circularized DNA probe carrying the imprinted information from the interrogated region (step 2). In this way the whole region was uniquely interrogated by a single MIP which contained the methylation information from the region of interest. Finally, the MIP circularized product was amplified by asymmetric PCR to generate a single stranded-amplicon which was captured by a receptor oligo sequence previously immobilized onto the SPR sensing surface (step 3). For interrogating the methylation information carried by the captured PCR amplicons, a synthetic reading ss-oligo was specifically hybridized to the region of interest contained in the MIP amplicon sequence. Since this reading oligo was designed to specifically match the methylated epigenotype, hybridization could only take place on PCR amplicons generated from methylated DNA templates. When monitoring the SPR signal generated with the successful hybridization of reading oligo, there was a clear correlation between the presence of the methylated amplicon and increase of the SPR signal (inset in Scheme 1). The presence of methylated amplicon increases the mass on the SPR sensing layer thereby increasing SPR signal.

Prior to detecting cytosine methylation in DNA derived from $\mathrm{MCF}_{7}$ cell line and WGA, we tested the applicability of this sensing scheme with short synthetic DNA sequences mimicking the methylated $\left(\mathrm{S}-\mathrm{MIP}_{\mathrm{Meth}}\right)$ and unmethylated (S-MIP Unmeth) epigenotypes (Table S1, ESI + ). Standard goldthiol chemistry was used to immobilize $1 \mu \mathrm{M}$ thiolated capture probe in the presence of $100 \mathrm{nM}$ mercaptohexanol $(\mathrm{MCH})$ onto the SPR sensing surface (Figure 1, condition I). MCH was employed as backfiller to prevent nonspecific adsorption on gold surface and to increase hybridization efficiency. ${ }^{21}$ Since both methylated and unmethylated targets had the same receptor matching sequence, they should be equivalently captured on the sensor surface. However, unmethylated targets generated relatively larger signals compared to that of the methylated one. A possible explanation for this enhanced response would be the larger nonspecific adsorption of adenine-enriched unmethylated target onto the gold surface (adenine bases are reported to have a large affinity towards gold ${ }^{22}$ ). We also believe that the presence of secondary structures (hairpins) in the targets could have a contribution towards this nonspecific adsorption, since the receptor-matching region would be less available for hybridization. The larger signal for $300 \mathrm{nM}$ reading oligo hybridized with $200 \mathrm{nM}$ unmethylated target (Condition I, Bar in aquamarine) further highlighted that a significant bias towards the unmethylated target during the reading oligo step might be introduced. In order to avoid these problems, we used (i) a monolayer with the high MCH loading as backfiller, and (ii) helper oligonucleotides, ${ }^{23}$ i.e. short ss-oligos partially matching with target sequences (Fig. S2, ESI ${ }^{\dagger}$ for details) to unfold its secondary structure. As can be seen in Fig 1 (Bars in Condition II), the combined action of extra $\mathrm{MCH}$ loading and addition of helpers to the targets increased the SPR signals for both the methylated and unmethylated targets compared to those obtained under Condition I. This is probably due to the more availability for receptor matching region for the hybridization with surface bound thiolated DNA. It is also noted that the signal for the reading oligo with unmethylated target was reduced significantly (i.e., almost similar to background level) while that for the methylated target increased slightly. This is probably due to the combined effect of helpers and $\mathrm{MCH}$ backfilling where $\mathrm{MCH}$ reduced non-specific adsorption on gold surface and helper oligos increased the specificity of the hybridization.
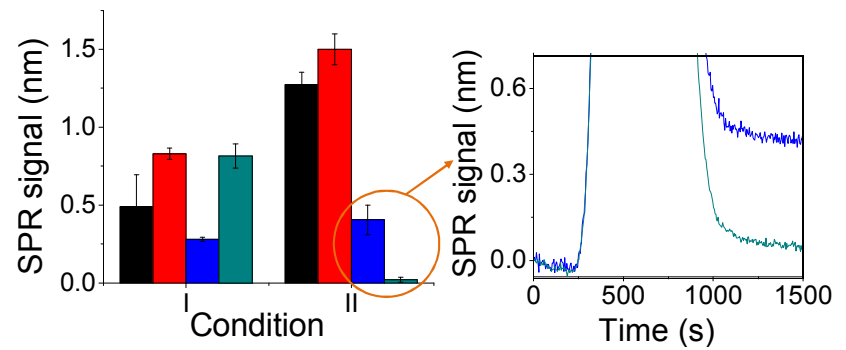

Fig. 1. SPR responses for the capture of $200 \mathrm{nM}$ synthetic methylated (black) and $200 \mathrm{nM}$ unmethylated (red) targets followed by their detection with $300 \mathrm{nM}$ synthetic reading oligo (blue for methylated targets; aquamarine for unmethylated targets). The self-assembled monolayer of the thiolated capture probe was formed via (Condition I) ex-situ immobilisation of $1 \mu \mathrm{M}$ thiolated DNA $+100 \mathrm{nM}$ aqueous $\mathrm{MCH}$ and (Condition II) condition I + in-situ immobilisation of $1 \mathrm{mM}$ aqueous $\mathrm{MCH}$ solution + capture of synthetic targets pre-treated with helper oligos. Right Panel: SPR sensorgrams of the reading oligos hybridized with methylated (blue) and unmethylated (aquamarine) targets. Each data point in left Panel represents the average of three separate trials $(n=3)$, and error bars represent standard deviation of measurements within each experiments. Relative standard deviation was found to be $<7.0 \%(n=3)$.

To evaluate the detection limit of our approach, we measured SPR signal for the capture of 100 to $400 \mathrm{nM}$ of the synthetic methylated targets (black Bars in Fig. 2A). Each concentration measurement was followed by a 300-500 nM reading oligo measurement step (blue bars in Fig 2A). Clearly, the SPR signal was a function of concentration of synthetic methylated targets. The minimum detectable concentration for methylated target was $100 \mathrm{nM}$. It is noted that the SPR signal for the reading oligo was also increased with increasing the concentration of methylated targets. This is because larger number of methylated sequences captured (as a function of concentration of target) onto sensing surface could hybridised with higher amount of reading oligo, and hence enhanced SPR signal was observed.

The specificity of our assay was tested against regional methylated target in the presence of an excess of unmethylated target (Fig. 2B). Clearly, $100 \mathrm{nM}$ methylated targets can easily be detected in the presence 
of $300 \mathrm{nM}$ of unmethylated target (this sample mixture corresponds to $25 \%$ methylation). This level of specificity indicates that our method could be applicable for epigenetic studies on biological samples such as samples containing a heterogeneous mixture of tumor and adjacent normal cells. Additionally, since the response from the $25 \%$ methylated target was significantly higher than that of $0 \%$ methylation (Fig. $2 \mathrm{~B}$ ), we estimated

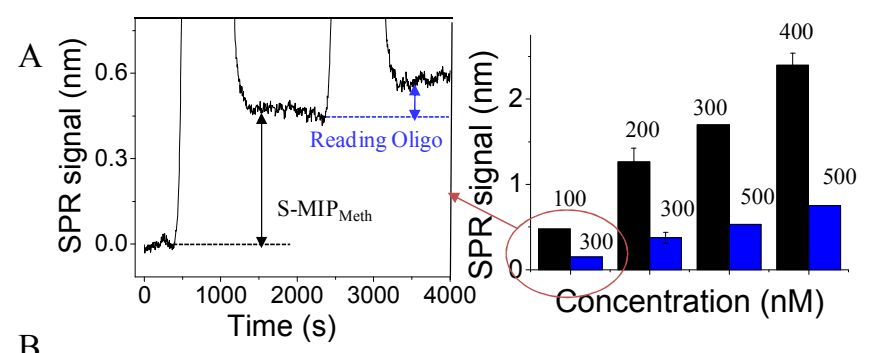

$\mathrm{B}$

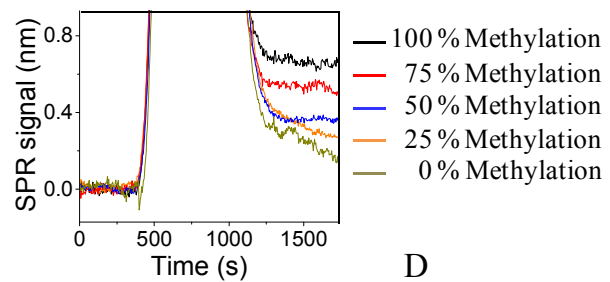

$\mathrm{C}$
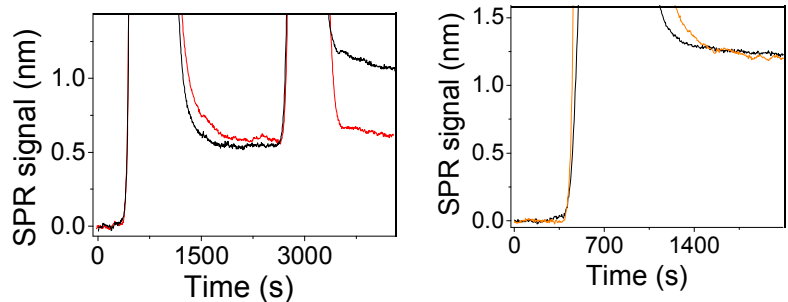

that our approach was sensitive at least for $25 \%$ methylation changes.

Fig. 2. (A) SPR sensorgram for $100 \mathrm{nM}$ synthetic methylated target (S-MIP $\mathrm{Meth}_{\text {) }}$ capture followed by $300 \mathrm{nM}$ reading oligo step onto the sensor surface. Dependence of the SPR signals on the concentration of S-MIP Meth is showed in the right panel. (B) SPR sensorgrams obtained at 100, 75, 50, 25 and $0 \%$ methylation. The samples were made via mixing 400, 300, 200, 100 and $0 \mathrm{nM}$ SMIP $_{\text {Meth }}$ target with $0,100,200,300$ and $400 \mathrm{nM}$ synthetic unmethylated target (S-MIP Unmeth), respectively. (C) SPR sensorgram for capturing and reading of the EN1 region from (i) $30 \mu \mathrm{L}$ MCF7 derived (i.e., methylated, black) and WGA derived (i.e., unmethylated, red) MIPs amplicons, and (D) $1^{\text {st }}$ (black) and $70^{\text {th }}$ (orange) measurements for $200 \mathrm{nM}$ synthetic methylated target.

We next demonstrated the applicability of our methodology for the reading of DNA methylation in a biological extract by performing the assay on breast cancer MCF7 cell line DNA. To avoid any potential bias towards a particular epigenotype, the target $\mathrm{EN}_{1}$ copy number in DNA from MCF7 and WGA DNA were normalized prior to MIP assay. This assay required only 50 $\mathrm{ng}$ of bisulfite treated DNA and $1 \mathrm{pg}$ (22 attomoles) of MIPs which generated $60 \mu \mathrm{L}$ of MIP derived ss-amplicons. We found that $30 \mu \mathrm{L}$ of these amplicons spiked in $200 \mu \mathrm{L}$ of buffer solution containing the oligonucleotide helpers were sufficient to obtain a significant detectable signal with the reading oligo (Fig. $2 \mathrm{C}$ ). The average reading signal for the amplicons from MCF7 cells was about 15-times larger than that of those from WGA DNA, indicating that the target region in MCF7 cells were methylated. The sensitivity and specificity of signals obtained for the reading of $30 \mu \mathrm{L}$ of MIP amplicon was comparable to that obtained for $200 \mathrm{nM}$ synthetic targets.

Finally, reusability of the receptor monolayer was examined by comparing SPR signals generated for $200 \mathrm{nM}$ synthetic methylated target over seventy $(n=70)$ independent measurements on the same monolayer surface. The monolayer was regenerated by using $50 \%$ formamide aqueous solution in-between two successive measurements. Fig. 2D shows that monolayer can be regenerated without losing any capture performance over this long period of SPR measurements. This feature of our approach could be very useful for diagnostic applications where routine monitoring of different patient's samples could be addressed using the same sensor chip, allowing it to be a simpler, faster, robust and inexpensive alternative to traditional detection methodologies.

In summary, we have demonstrated a rapid, label-free and real-time detection of regional DNA methylation. The combined use of MIP and SPR detection facilitates three critical improvements to the traditional DNA methylation detection methods that include: (i) real-time and label-free detection giving instant read-outs by avoiding artifacts generally associated with the use of labels, (ii) cost-effective approach requiring minimal operational requirements (e.g., syringe pump and custom made SPR setup), and (iii) potential multiplexing capability of MIPs and an multichanneled SPR platform which can facilitate high throughput analysis. Although further validation needs to be done to examine variation in methylation in clinically relevant samples, we believe that these unique features along with the high specificity, sensitivity, and ability to detect a low percentage of methylation in presence of large excess of unmethylated DNA, will make this strategy very appealing for diagnostic purposes.

This work was supported by the UO fellowship (2012001456) awarded for LGC, ARC DECRA (DE120102503) awarded for MJAS, and National Breast Cancer Foundation (NBCF) of Australia (CG-08-07 and CG-12-07) awarded for MT. The fabrication work was performed at Queensland node of the Australian National Fabrication Facility (Q-ANFF).

\section{Notes and references}

1. M. Suzuki and A. Bird, Nat. Rev. Genet., 2008, 9, 465-476.

2. H. Heyn and M. Esteller, Nat. Rev. Genet., 2012, 13, 10, 679-692.

3. K. M. Koo, et al., Biosens. Bioelectron., 2014, in press.

4. P. Wang, et al., Biosens. Bioelectron. 2013, 45, 34-39.

5. N. Pälmke, et al., Methods Mol. Biol., 2011, 53, 175-184.

6. Y. Yu, et al., Anal Chem., 2010, 82, 12, 5012-5019.

7. P. Wang, et al., Chem. Commun. 2012, 48, 10754-10756.

8. J. D. Suter, et al., Biosens. Bioelectron., 2010, 26, 1016-1020.

9. S. Pan, et al., Biosens Bioelectron., 2010 26, 850-853.

10. S. Fouse, et al., Epigenomics, 2010, 2, 105-117.

11. L. G. Carrascosa, et al., Anal. Bioanal. Chem., 2009, 393, 1173-1182.

12. J. Homola, Chem. Rev., 2008, 108, 462-493.

13. S. Nair, et al., Epigenetics, 2011, 6, 34-44.

14. M. Nilsson, et al., Science, 1994, 265, 2085-2088.

15. Y. Wang, et al., Cancer Genet. Cytogenet., 2012, 205, 341-355.

16. G. J. Porreca, et al., Nat. Methods, 2007, 4, 931-936.

17. J. Deng, et al., Nat. Biotechnol., 2009, 27, 353-360.

18. R. Palanisamy, et al., Anal. Chem., 2011, 83, 2631-2637.

19. T. Rauch, et al., Pro. Natl. Acad. Sci. 2007, 104, 5527-5532.

20. S. Hughes and J. Jones, BMC Mol. Biol., 2007, 16, 8:91 (1-7).

21. T. M. Herne and M. J. Tarlov, J. Am. Chem. Soc., 1997, 119, 89168920.

22. H. Kimura-Suda et al., J. Am. Chem. Soc., 2003, 125, 9014-9015.

23. J. T. Kindt and R. C. Bailey, Anal. Chem., 2012, 84, 8067-8074. 


\section{ChemComm}

\section{Accepted Manuscript}
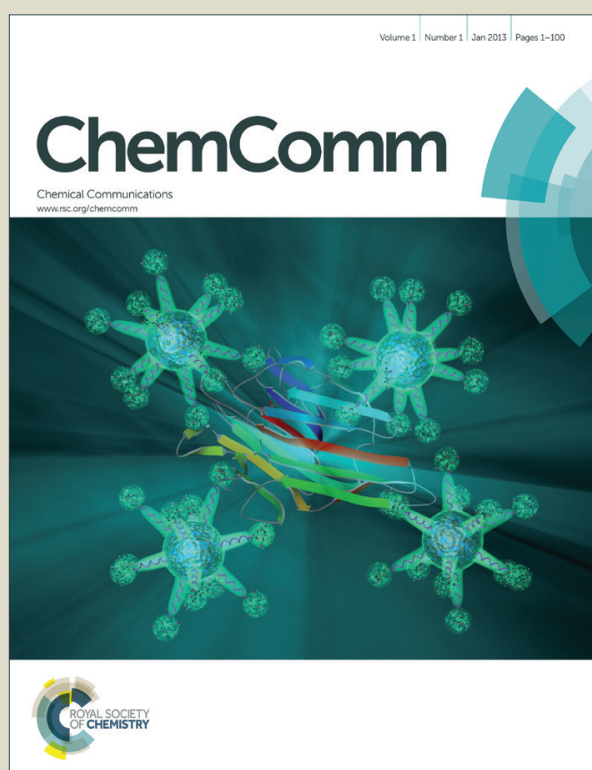

This is an Accepted Manuscript, which has been through the Royal Society of Chemistry peer review process and has been accepted for publication.

Accepted Manuscripts are published online shortly after acceptance, before technical editing, formatting and proof reading. Using this free service, authors can make their results available to the community, in citable form, before we publish the edited article. We will replace this Accepted Manuscript with the edited and formatted Advance Article as soon as it is available.

You can find more information about Accepted Manuscripts in the Information for Authors.

Please note that technical editing may introduce minor changes to the text and/or graphics, which may alter content. The journal's standard Terms \& Conditions and the Ethical guidelines still apply. In no event shall the Royal Society of Chemistry be held responsible for any errors or omissions in this Accepted Manuscript or any consequences arising from the use of any information it contains. 